\title{
Calcium Control of Endocytic Capacity at a CNS Synapse
}

\author{
J. Balaji, ${ }^{1}$ Moritz Armbruster, ${ }^{2}$ and Timothy A. Ryan ${ }^{1}$ \\ ${ }^{1}$ Department of Biochemistry, Weill Cornell Medical College, and ${ }^{2}$ David Rockefeller Graduate Program of The Rockefeller University, New York, New York \\ 10065
}

The ability to recycle synaptic vesicles is a crucial property of nerve terminals that allows maintenance of synaptic transmission. Using high-sensitivity optical approaches at hippocampal nerve terminals in dissociated neurons in culture, we show that modulation of endocytosis can be achieved by expansion of the endocytic capacity. Our experiments indicate that the endocytic capacity, the maximum number of synaptic vesicles that can be internalized in parallel at individual synapses, is tightly controlled by intracellular calcium levels. Increasing levels of intracellular calcium, which occurs as firing frequency increases, significantly increases the endocytic capacity. At physiological temperature after $30 \mathrm{~Hz}$ firing, these synapses are capable of endocytosing at least $\sim 28$ vesicles in parallel, each with a time constant of $\sim 6 \mathrm{~s}$. This calcium-dependent control of endocytic capacity reveals a potentially useful adaptive response to high-frequency activity to increase endocytic rates under conditions of vesicle pool depletion.

Key words: endocytosis; synapse; pHluorin; recycling; calcium; vesicles

\section{Introduction}

After neurotransmitter release at nerve terminals, synaptic vesicle components are recycled for further rounds of reuse. The speed of this process in part determines how quickly vesicle pools become depleted during activity, which in turn impacts synaptic efficacy. We recently demonstrated that endocytosis after single vesicle fusion is a stochastic process with a mean retrieval time of $\sim 14$ s (Balaji and Ryan, 2007) at small synapses in the CNS, and others have recently shown that retrieval for modest stimulation appears to operate via clathrin-mediated endocytosis (Granseth et al., 2006). During repetitive stimulation, the ability to assemble multiple clathrin-coated pits will be necessary to provide efficient vesicle recycling unless other mechanisms of retrieval become operational. The number of such sites that can operate in parallel will effectively determine the endocytic capacity of the synapse in question. In our original observations using synaptopHluorins (spH) (Sankaranarayanan and Ryan, 2000), we discovered that, with sufficient stimulation, endocytosis operates at a constant rate, suggesting that the synapse was operating under conditions in which this capacity was saturated. Improvements in measurement sensitivity based on tagging alternate synaptic vesicle proteins that have lower surface expression showed that the characteristic endocytic retrieval time remains invariant at $\sim 14$ s for up to 40 action potential (AP) stimuli (Granseth et al., 2006), suggesting that for this level of stimulation, all vesicles undergoing exocytosis are endocytosed in parallel, with each vesicle having a

Received March 12, 2008; revised May 5, 2008; accepted May 20, 2008.

This work was supported by grants from the National Institutes of Health (T.A.R.) as well as the David Rockefeller Graduate School of The Rockefeller University. We thank members of the Ryan Laboratory for useful discussions and Ricky Kwan for excellent technical assistance. vGlut-pHluorin was kindly provided by Susan Vogelmeier and Robert Edwards.

Correspondence should be addressed to Timothy A. Ryan, Department of Biochemistry, Weill Cornell Medical College, 1300 York Avenue, Room E-107, New York, NY 10065. E-mail: taryan@med.cornell.edu.

DOI:10.1523/JNEUROSCI.1082-08.2008

Copyright $\odot 2008$ Society for Neuroscience $\quad$ 0270-6474/08/286742-08\$15.00/0 mean endocytosis time of $\sim 14 \mathrm{~s}$. We took advantage of recent improvements in optical collection efficiencies in our measurements (Balaji and Ryan, 2007) to examine the question of how endocytosis speed depends on the amount of surface accumulation of synaptic components by examining this for a large number of trials over a wide range of stimulation (from 5 to $500 \mathrm{AP}$ ) at each synapse. This approach shows that the time constant for endocytosis is invariant below a critical level of accumulation of synaptic vesicle components, but begins to slow only above this threshold.

Previous studies have shown that two variables that modulate endocytosis at synapses are intracellular calcium levels and temperature. We show here that the endocytic capacity is dynamically modulated by increases in intracellular calcium but that the fundamental time constant for each vesicle is relatively insensitive to intracellular calcium levels. Elevation of the temperature to $36^{\circ} \mathrm{C}$ in contrast results in a significant increase in the speed of endocytosis of each vesicle. These measurements reveal that calcium plays a critical role in the adaptive response of the synapse to increased endocytic loads, and reveals two distinct properties of the endocytic machinery that serve as potential substrates for modulation.

\section{Materials and Methods}

Cell culture. Hippocampal CA3-CA1 regions were dissected from 1- to 3-d-old Sprague Dawley rats, dissociated, and plated onto polyornithine-coated glass for 14-25 d as previously described (Ryan and Smith, 1995). vGlut1-pHluorin/synaptopHluorin was transfected 6-8 d after plating. Experiments were performed $14-25 \mathrm{~d}$ after plating $(8-18 \mathrm{~d}$ after transfection), and the coverslips were mounted in a rapidswitching, laminar-flow perfusion and stimulation chamber (volume $\sim 75 \mu \mathrm{l}$ ) on the stage of a custom-built laser illuminated epifluorescence microscope or a custom-built laser-scanning microscope. Images were acquired with Andor iXon + (Model \# DU-897E-BV) back-illuminated EMCCD camera in the epifluorescence microscope or using customwritten control software for LSM. Action potentials were evoked by pass- 
ing $1 \mathrm{~ms}$ current pulses, yielding fields of $\sim 10$ V/cm via platinum-iridium electrodes. Cells were continuously perfused $(1-1.5 \mathrm{ml} / \mathrm{min}$, $\sim 25^{\circ} \mathrm{C}$ ) in a saline solution containing (in $\mathrm{mm}$ ) $119 \mathrm{NaCl}, 2.5 \mathrm{KCl}, 2 \mathrm{CaCl}_{2}, 2 \mathrm{MgCl}_{2}, 25$ HEPES (buffered to $\mathrm{pH} 7.4$ ), 30 glucose, $10 \mu \mathrm{M}$ CNQX (6-cyano-7-nitroquinoxaline-2,3-dione) (Research Biochemicals), and $50 \mu \mathrm{M}$ AP5 (D,L-2amino-5-phosphonovaleric acid) (Research Biochemicals). When lower $\left[\mathrm{CaCl}_{2}\right]$ was used, $\left[\mathrm{MgCl}_{2}\right]$ was increased to keep the divalent ion concentration constant. Unless otherwise noted, all chemicals were obtained from Sigma. Physiological temperature experiments were done with a custom-built proportional current controller heating the microscope objective. The objective was maintained at $36^{\circ} \mathrm{C}$, with the sample being within $\sim 1^{\circ} \mathrm{C}$ of the objective. For physiological temperature experiments, the same saline solution was buffered to $\mathrm{pH} 7.4$ at $36^{\circ} \mathrm{C}$ and continuously perfused at $200-400$ $\mu \mathrm{l} / \mathrm{min}$.

Calcium dye measurements. The AM esters of Fluo-3 or Mag Green (Invitrogen) were added to get a final concentration of $10 \mu \mathrm{M}$. After a $15 \mathrm{~min}$ incubation, the dye was washed off for 10 $\mathrm{min}$, and then the response was recorded using a laser-scanning microscope in the line scanning mode. Presynaptic varicosities were chosen for line-scan imaging. The fractional change in fluorescence at steady state is measured as a function of different frequencies at $2 \mathrm{~mm}$ external calcium and at $10 \mathrm{~Hz}$ stimulation at $1-3 \mathrm{~mm}$ external calcium. These fractional changes were measured for both Fluo- 3 and Mag Green to ensure that the measurements are not obscured by low sensitivity (Mag Green) or dye saturation (Fluo-3).

Optical setup. Measurements were made either using a custom-built laser-scanning confocal microscope based on Zeiss Axiovert 200 modified to use either a GaAsP photomultiplier module (H7422-40; Hamamatsu) as a non-descanned detector for enhanced sensitivity or by epifluorescence microscopy. In the latter case, we used laser illumination $\left(<0.5 \mu \mathrm{W} / \mu \mathrm{m}^{2}, 488 \mathrm{~nm}\right)$ with the beam sufficiently expanded to get near uniform illumination, and images were collected with an Andor iXon+ (Model \# DU-897E-BV) back-illuminated EMCCD camera. $\mathrm{Ar}^{+}$ ion laser or a solid-state diode-pumped $488 \mathrm{~nm}$ laser that was shuttered using acousto-optic modulation in all periods during which we were not acquiring data served as a common light source for both setups. Fluorescence excitation and collection was done through a $40 \times 1.3$ numerical aperture Fluar Zeiss objective using 515-560 nm emission and $510 \mathrm{~nm}$ dichroic filters.

Image and data analysis. Images were analyzed in ImageJ (http://rsb. info.nih.gov/ij/) using a custom-written plugin (http://rsb.info. nih.gov/ij/plugins/time-series.html). All functionally visible varicosities were selected for analysis by testing their responsiveness to multiple (3-4) rounds of test stimuli delivered as $6 \mathrm{AP}$ trains at $33 \mathrm{~Hz}$. The fluorescence intensity within a circular region of interest of $1.25 \mu \mathrm{m}$ radius were averaged together for each bouton, which avoided fluorescence decay caused by faster diffusive processes (Granseth et al., 2006). Furthermore, it can be seen from Equations 2 and 7 of the Appendix that for $t>\tau_{r}$, the contribution from reacidification is negligibly small, and the fluorescence decay $[F(t)$ in Eq. 7] reduces to a single exponential. The later part of the ensemble average response $(t>$ stimulus time or $4 \mathrm{~s}$ for stimulus time $<4 \mathrm{~s}$ ) was fit to an exponential. For analysis of the endocytic capacity, the endocytic constants in the presaturated region (see Results) are used for normalization. Stimulation for $10 \mathrm{~s}$ at $33 \mathrm{~Hz}$ was used to estimate the total recycling vesicle pool size in each experiment. We previously showed that the peak accumulation for such experiments is $\sim 85 \%$ of the total recycling pool measured using bafilomycin (Fernandez-Alfonso and Ryan, 2004). Fig. S1 A (available at www. jneurosci.org as supplemental material) shows the average time constants used to normalize various runs. In general, we find that there is slightly greater heterogeneity across cells in the value of $\tau_{0}$ and a slightly larger value in $\mathrm{spH}$ cells compared with vGlut-pHluorin (vGpH)transfected cells. We suspect that this difference might arise from the tendency of our $\mathrm{spH}$ vector to express more frequently in GABAergic cells than the $\mathrm{vGpH}$ vector.

The cumulative exocytosis curve during a sustained stimulation (see Fig. $3 C$ ) is constructed by summing the instantaneous fluorescence changes associated with each stimulus. The mean fluorescence curve is constructed by taking the instantaneous fluorescence one time point before the arrival of the stimulus. The apparent endocytosis curve is constructed by subtracting the fluorescence curve from the exocytosis curve point by point.

\section{Results}

\section{Following endocytosis using pHluorins after small and prolonged stimulus}

To examine the endocytic behavior as a function of the accumulation of vesicle proteins on the synaptic surface, we made use of quantitative measurements of dynamic behavior of pHluorintagged vesicle proteins after AP firing. pHluorins are a variant of GFP whose fluorescence is quenched by proton binding with a pKa of $\sim 7.1$ (Sankaranarayanan et al., 2000). When targeted to the lumen of a synaptic vesicle, the fluorescence is quenched, and after exocytosis and exposure to the more alkaline environment of the extracellular milieu, the quenching is relieved, resulting in an $\sim 25$-fold increase in fluorescence. During continuous AP firing, the fluorescence at any given time corresponds to a net balance of exocytosis and endocytosis (Sankaranarayanan and Ryan, 2000; Fernandez-Alfonso and Ryan, 2004). The net fluorescence change reflects the accumulation of pHluorin-tagged protein on the synaptic surface; because the time constant for reacidification is $\sim 4$ s (Atluri and Ryan, 2006; Granseth et al., 2006), the contribution from recently endocytosed and alkaline vesicles is minimal. After stimulation, the rate of decay of fluorescence provides a good measure of the rate of endocytosis. We used this property to examine how endocytosis varied as a function of the amount of accumulation on the synaptic surface, by examining the fluorescence responses to varied lengths of stimulus at constant firing frequency. Figure 1 shows an example of the ensemble response from a population of synaptic boutons from either $\mathrm{spH}$ transfected (Fig. $1 A$ ) or vGpH-transfected (Fig. $1 B$ ) hippocampal neurons stimulated for varied duration at $10 \mathrm{~Hz}$. In each case the fluorescence reached different peak levels depending on the duration of action potential firing. The degree of accumulation 
A

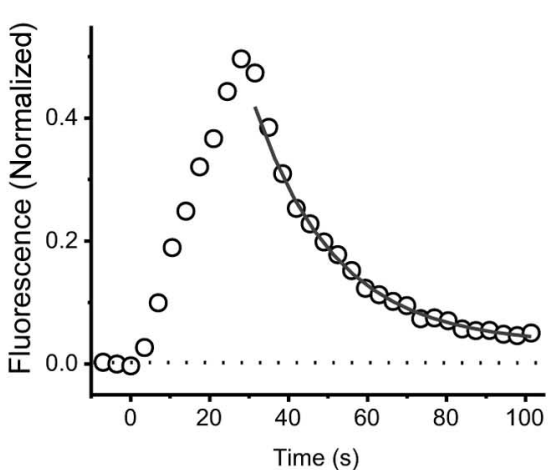

C

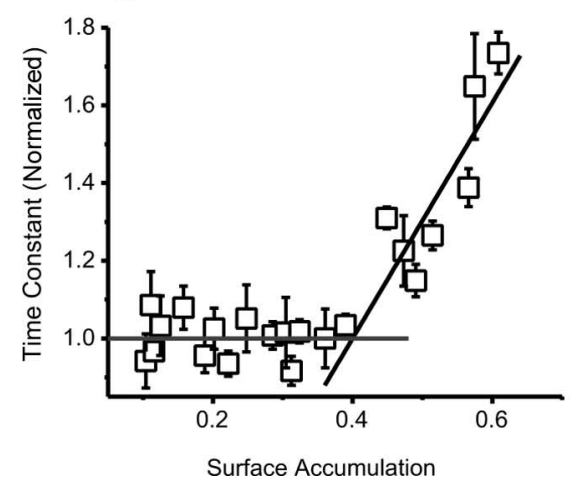

B

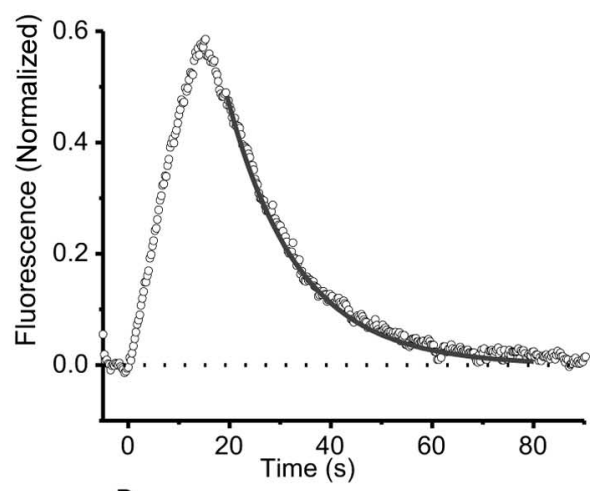

D

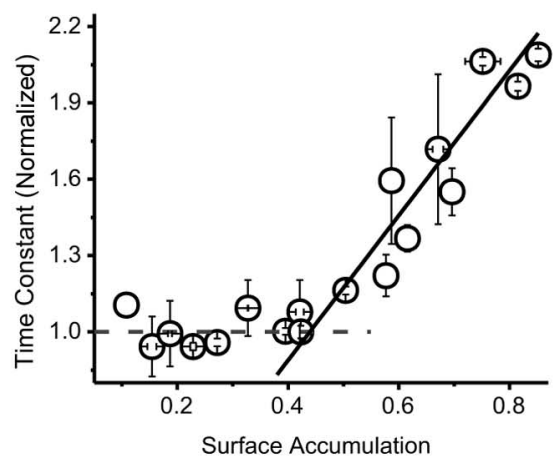

Figure 2. Poststimulus decay in pHluorin response fits well to single-exponential decay. $A, B$, Open circles are the average fluorescent traces of boutons transfected with $\mathrm{spH}$ and $\mathrm{vGpH}$, respectively ( $\sim 30$ boutons in each case). The line is the exponential fit to the data. $\boldsymbol{C}, \boldsymbol{D}$, The time constants for synaptopHluorin- ( $n=3$ cells, $45-60$ boutons each) and vGpH- ( $n=4$ cells, $30-50$ boutons each) transfected neurons measured as a function of surface accumulation. The decay constants start to increase after $\sim 45 \%$ for each pHluorin-tagged synaptic vesicle protein. The increasing decay constants fit to a straight line. The point at which this line intersects the fundamental time constant (normalized to unity) yields the $C_{\text {endo }}$ of the cell. For $\operatorname{spH}(\boldsymbol{C})$, the mean time constant in the nonsaturating region is $19.5 \pm 1.3 \mathrm{~s}$, and for $\mathrm{vGpH}(\boldsymbol{D})$, it is $14.2 \pm 2.2 \mathrm{~s}$.

can be normalized in each experiment to the peak reached during a $10 \mathrm{~s}$ burst at $33 \mathrm{~Hz}$ (Fig. 1), which we previously showed leads to accumulation of $\sim 85 \%$ of the entire recycling pool (FernandezAlfonso and Ryan, 2004). Examination of the poststimulus fluorescence decay for both $\mathrm{vGpH}$ and $\mathrm{spH}$ responses shows a progressive acceleration in endocytic rate until a given accumulation, beyond which the endocytotic speed reaches a limiting value, suggesting a possible saturation of the endocytic machinery.

\section{Determination of maximal vesicle number endocytosed with invariant kinetics}

To characterize the point at which these nerve terminals can no longer endocytose vesicles with equal efficiency, we examined the variation of the decay constant $(\tau)$ of the poststimulus fluorescence recovery, as a function of the total surface accumulation of the reporter protein. The accumulation is expressed as the fraction of the entire recycling pool, as determined from the highfrequency response peak (see Materials and Methods). We reasoned that in the invariant regime, endocytosis should proceed as a first- or pseudo-first-order process with respect to vesicles, reflecting the ability of the synapse to endocytose all of the synaptic vesicles in parallel with equal efficacy. The time constant would start to increase once the endocytic capacity of the synapse is exceeded. The fluorescence decays for each run were well fit to a single exponential (Fig. $2 A, B$ ), providing a measure of $\tau$. The fluorescence from the poststimulus $\left(t>t_{\text {stimulus }}+4 \mathrm{~s}\right)$ region for the fit was chosen to minimize the contribution from reacidifica- tion (see Materials and Methods). For lower accumulations, the value of $\tau$ was invariant, in agreement with recent measurements using pHluorin-tagged synaptophysin (Granseth et al., 2006). To measure the degree to which endocytosis slowed as a function of accumulation in different cells, we normalized the values of $\tau$ to the average value in the invariant region (stimuli of $<100 \mathrm{AP}$ ) in each experiment. This allows us to bring out any phenomenon that is obscured by the small intrinsic variability from cell to cell of the nonsaturating value of $\tau$. A plot of the normalized $\tau$ values for many experiments with $10 \mathrm{~Hz}$ stimulation as a function of surface accumulation (Fig. $2 C, D)$ shows two clearly different regimes: below $\sim 45 \%$ surface accumulation, $\tau$ was independent of accumulation, but above this value, it increased linearly. This suggests the existence of saturable endocytosis as hypothesized before (Sankaranarayanan and Ryan, 2000). Both spH (Fig. 2C) and vGpH (Fig. 2D) showed that the saturation occurs at similar surface accumulation levels. These experiments indicate that there are two distinct operational regimes for endocytosis. We quantified the transition between these two regimes to a first approximation as the point on the abscissa where the straight line fit to the saturation regime is unity. It should be noted that this approach to defining the transition is only for convenience, because the actual transition is likely to be more gradual, as would be expected of any pseudo-first-order reaction. We operationally defined this transition point expressed in terms of the fraction of the total recycling pool of synaptic vesicles as the endocytic capacity, $C_{\text {endo }}$, of the synapse. We refer to the invariant time constant in the nonsaturating regime as the fundamental time constant, $\tau_{0}$.

\section{The time constant is invariant during repetitive stimulation at low frequencies}

Our data suggest that one of the critical variables determining the speed of endocytosis is the net accumulation of synaptic components on the cell surface, and if this accumulation is lower than the endocytic capacity, endocytosis will proceed with a time constant of $\sim 14 \mathrm{~s}$. Thus, even during persistent activity, as long as accumulation of synaptic components remains low, the mean time constant for endocytosis should remain unchanged. Recently we demonstrated that $\mathrm{vGpH}$ recordings provide accurate measures of exocytosis at the single AP level (Balaji and Ryan, 2007). We took advantage of this ability to continuously monitor exocytosis as well as the total average fluorescence resulting from accumulating $\mathrm{vGpH}$ on the cell surface to determine the average time constant for endocytosis during repetitive stimulation. During persistent activity at individual boutons, the ongoing endocytic retrieval tries to balance the exocytic delivery of new synaptic vesicle protein with each stimulus (Fig. $3 A$ ). The ensemble average across many boutons provides a very robust measure of the average amount of exocytosis with each stimulus (Balaji and Ryan, 2007) as well as the net accumulation of vGpH (Fig. 3B). 
The cumulative amount of exocytosis during repetitive stimulation can be obtained by summing the average fluorescence change for each action potential stimulus from the ensemble average (Fig. $3 C$ ). The total amount of endocytosis (Fig. 3 C) can be obtained by subtracting the average surface accumulation $([S])$ during the stimulus train from the cumulative exocytosis trace as well as a small correction factor to account for the contribution from the recently internalized alkaline pool (see Appendix). The slope of this cumulative endocytosis trace provides a measure of the rate of endocytosis $(V)$. This approach is similar to one in which we used bafilomycin to keep track of exocytosis independently of endocytosis using pHluorins (Sankaranarayanan and Ryan, 2001; Di Paolo et al., 2004; Schweizer and Ryan, 2006; Ferguson et al., 2007; Mani et al., 2007); however, here no extra pharmacological agents are needed, because the exocytosis transient is not contaminated by endocytosis, which can be monitored directly on a sufficiently fast time scale. In the nonsaturated regime, in which accumulation remains below the endocytic capacity, the rate of endocytosis should be proportional to the total amount of $\mathrm{vGpH}$ accumulated on the surface. Figure $3 D$ shows the results for the measured endocytic rate $V$ at 0.066 , 0.2 , and $0.5 \mathrm{~Hz}$ stimulation. If one normalizes these rates, however, to the amount of surface accumulation, one can estimate the rate constant, $k$, for each stimulus condition $(k=V /[S])$ (see Appendix). Measurements of $k$ were performed in this way for stimulus frequencies from 0.05 to $1 \mathrm{~Hz}$ and indicate that the apparent time constant for endocytosis $(1 / k)$ is approximately constant across this stimulus frequency range. Furthermore, the value of the time constant is in good agreement with that estimated from the poststimulus recovery in the nonsaturating regime (Fig. $2 B$ ), and comparison of the endocytic time trace obtained as described above during and after stimulation shows that they match closely (Fig. 4). These results all support the idea that at these nerve terminals, the time constant for endocytosis is not directly related to rates of exocytosis, but rather depends on whether or not one exceeds the endocytic capacity of the synapse.

\section{Elevations in intracellular calcium increase the saturable limit} of endocytosis

Previously, we showed that elevations in intracellular calcium accelerated the rate of endocytosis at these nerve terminals (Sankaranarayanan and Ryan, 2001). We therefore performed experiments to determine how this acceleration is achieved at these synapses. We measured the value of $\tau$ under conditions that lead to different internal calcium concentrations and determined how $\tau$ varied as a function of surface accumulation for these different units.
C

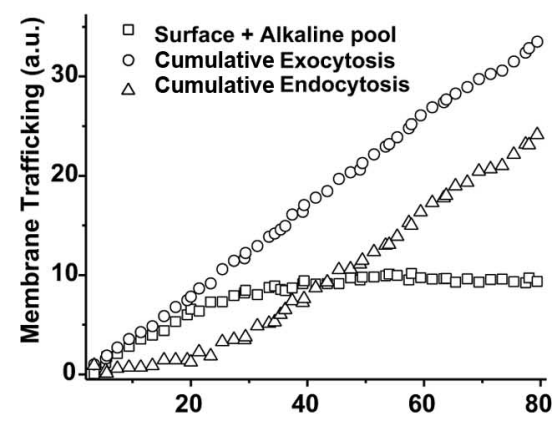

D

Time (s)
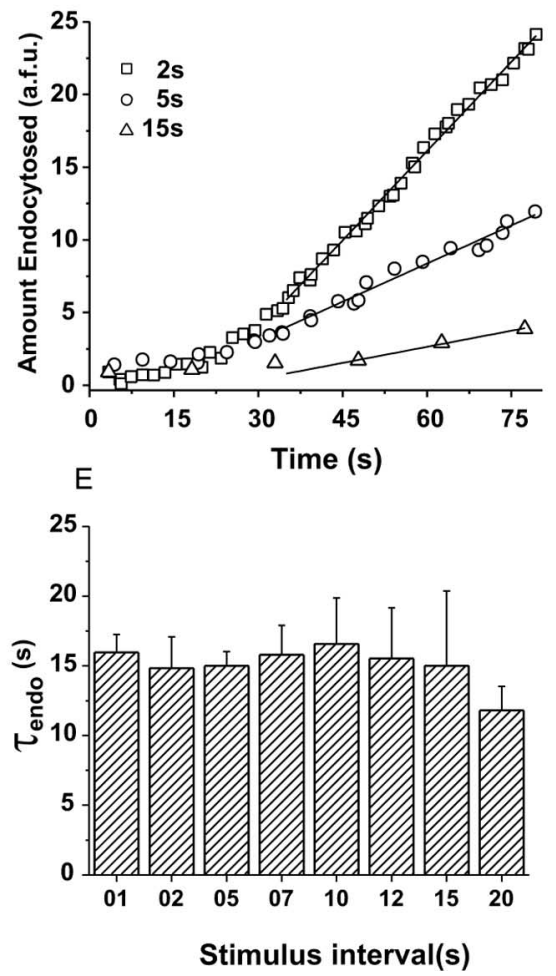

Stimulus interval(s)

Figure 3. Exocytosis and endocytosis during persistent stimulation followed in real time. $A$, Responses of three boutons for action potentials delivered every $15 \mathrm{~s}$ (arrows). $\boldsymbol{B}$, The ensemble average time trace obtained for stimuli delivered at 2,5, (the Appendix) for different stimulus intervals are shown (5 cells, 372 boutons). a.f.u., Arbitrary fluorescence units; a.u., arbitrary

conditions. We changed the internal calcium by two different approaches: (1) by changing the external calcium ion concentration $\left([\mathrm{Ca}]_{\mathrm{e}}\right)$ at a constant stimulus frequency and (2) by changing the stimulus frequency under the same external calcium concentration. Experiments using either spH (Fig. $5 A$ ) or vGpH (Fig. $5 B$ ) revealed that increasing the stimulus frequency shifts the point of saturation to the right, indicating an expansion in endocytic capacity, while leaving the fundamental time constant unchanged (see supplemental Fig. 1, available at www.jneurosci.org as supplemental material). Comparison of the variation of $\tau$ with surface accumulation for stimuli delivered in $[\mathrm{Ca}]_{\mathrm{e}}=1 \mathrm{~mm}$ with that for stimuli delivered in $[\mathrm{Ca}]_{\mathrm{e}}=3 \mathrm{~mm}$ also indicated clear 
A

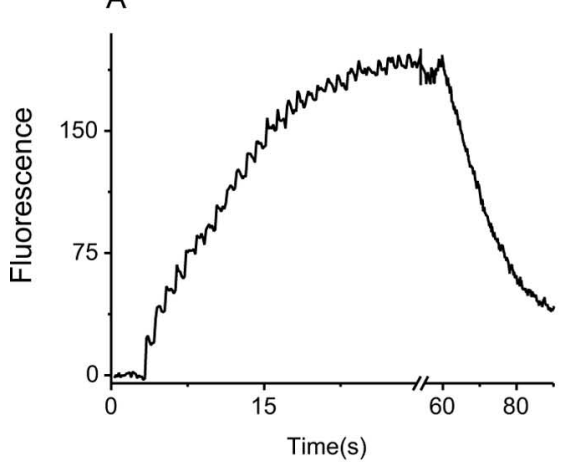

B

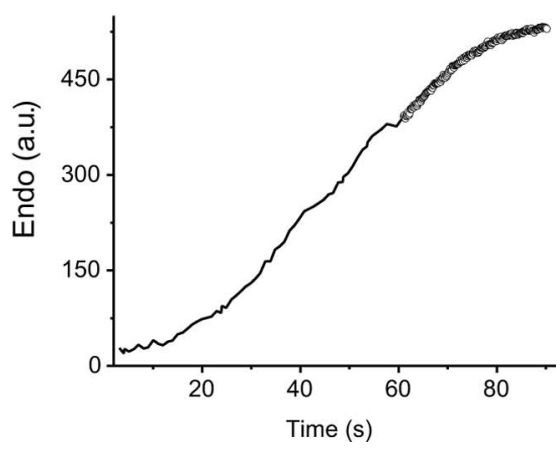

Figure 4. Endocytosis rate during and after stimulation is very similar. $\boldsymbol{A}$, The $\mathrm{vGpH}$ fluorescence curve during stimulation at $0.5 \mathrm{~Hz}$ is compared with the $\mathrm{vGpH}$ fluorescence trace obtained after the stimulus for the same set of synapses. $\boldsymbol{B}$, The derived cumulative endocytosis curve (black) during stimulation obtained from subtracting the surface accumulation from the cumulative exocytosis curve is very similar to the $\mathrm{vGpH}$ fluorescence decay (open symbols, here inverted for comparison), which is dominated by endocytosis after the stimulus. a.u., Arbitrary units.

A

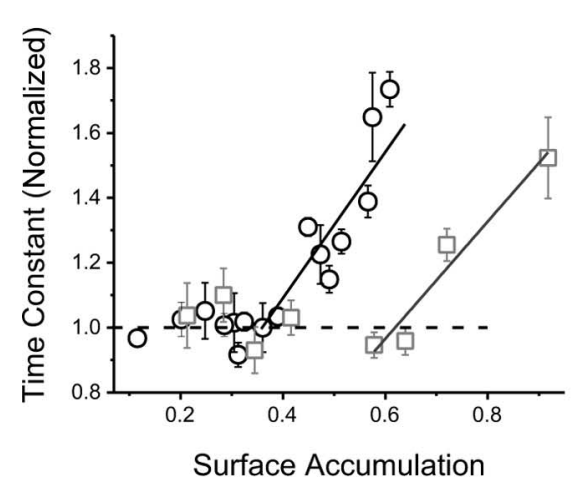

B

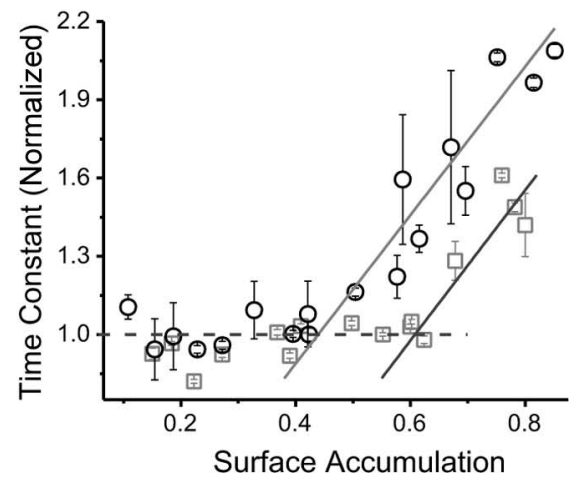

Figure 5. Endocytic capacity of the neuron is modulated by altering the levels of intracellular calcium. $\boldsymbol{A}, \boldsymbol{B}$, Plots of decay constants obtained for synaptopHluorin ( 5 cells) and vGpH (4 cells) obtained using varying stimulus duration $(6-50 \mathrm{~s})$ at $10 \mathrm{~Hz}$ (black circles) and $33 \mathrm{~Hz}$ (gray squares). $\boldsymbol{A}$, For $\mathrm{spH}$, the average time constant in the nonsaturating region is $19.5 \pm 1.3 \mathrm{~s}$ for the $10 \mathrm{~Hz}$ and $19.6 \pm 1.4 \mathrm{~s}$ for the $33 \mathrm{~Hz}$ data. $\boldsymbol{B}$, For $\mathrm{G} \mathrm{pH}$, the average time constant in the nonsaturating region is $14.2 \pm 2.2 \mathrm{~s}$ for the $10 \mathrm{~Hz}$ and $15.0 \pm 1.9 \mathrm{~s}$ for the $33 \mathrm{~Hz}$ data. Endocytosis saturates at higher accumulation, suggesting an increased endocytic capacity at higher frequency of stimulation. The "endocytic capacity" defined by abscissa of where the straight line fit (dashed line in both graphs) is unity.
A

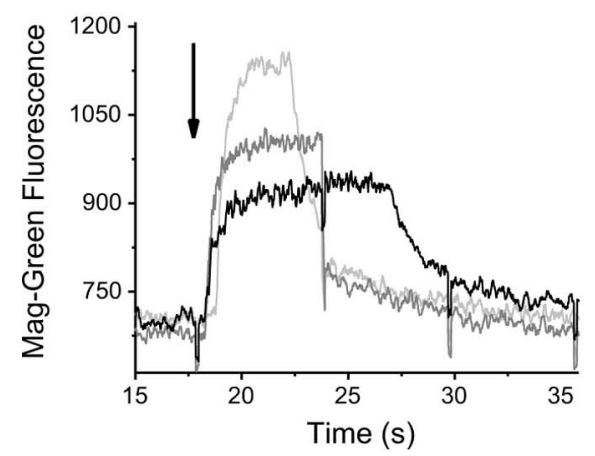

B

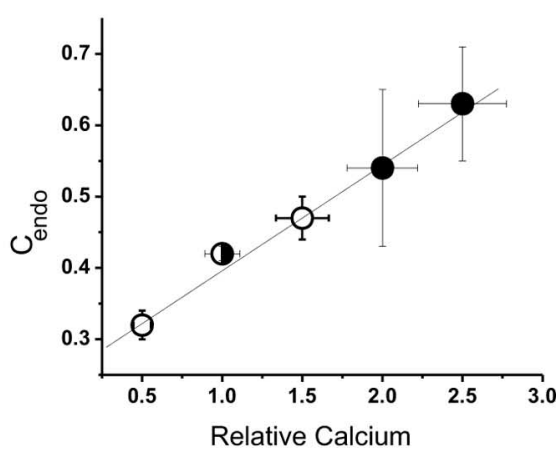

Figure 6. Endocytic capacity measured as a function of relative intracellular calcium. Fluorescent signal of calcium indicators (Mag Green) is used as a relative measure of intracellular steady-state calcium during the stimulus. $\boldsymbol{A}$, The fluorescence measured as a function of time for 10 (light gray), 20 (dark gray), and 30 (black) $\mathrm{Hz}$ stimulus at $2 \mathrm{~mm}$ external calcium measured at an individual synaptic varicosity. $\boldsymbol{B}$, Endocytic capacity, $\boldsymbol{C}_{\text {endo }}$ (circles), is plotted against the relative intracellular steady-state calcium levels obtained by changing either the stimulus frequency while keeping external $\mathrm{CaCl}_{2}$ at $2 \mathrm{~mm}$ (filled symbols, 10, 20, and $30 \mathrm{~Hz}$ ) or external calcium concentration while keeping the stimulus frequency at $10 \mathrm{~Hz}$ (open symbols, 1,2 , and $3 \mathrm{~mm}(\mathrm{CaCl})(8$ cells). shift in $C_{\text {endo }}$ toward higher values (see supplemental Fig. 2, available at www. jneurosci.org as supplemental material).

The onset of saturation appears to occur only for significant accumulation of synaptic vesicle components on the synaptic surface, which in turn occurs typically only after at least $100 \mathrm{AP}$ of stimulation at $10 \mathrm{~Hz}$ or above. We reasoned that the control of $C_{\text {endo }}$ by intracellular calcium was likely related to the average bulk cytoplasmic calcium levels reached during these stimulus periods (see Discussion). To correlate the value of $C_{\text {endo }}$ with intracellular calcium, we examined the relative intracellular calcium levels achieved for these different stimulus conditions by performing imaging experiments in synapses loaded with fluorescent indicator of calcium, Mag Green. Figure 6A shows that the Mag Green fluorescence increases and reaches near steady state after a few seconds during a prolonged stimulation. We performed experiments sequentially at the three stimulus frequencies used to manipulate $C_{\text {endo }}$ (Fig. 4) and determined the relative fluorescence increase with respect to the $10 \mathrm{~Hz}$ stimulation. Similar experiments were performed with sequential values of $[\mathrm{Ca}]_{e}$, and the fluorescence changes were normalized to the 10 $\mathrm{Hz},[\mathrm{Ca}]_{\mathrm{e}}=2 \mathrm{~mm}$ condition. Figure 6 shows the summary of the results comparing the value of $C_{\text {endo }}$ obtained for a given stimulus condition with the relative intracellular calcium ion level obtained in parallel experiments and shows a near linear dependence, supporting the view that the number of vesicles that can be internalized in parallel is under tight control by intracellular calcium. Furthermore, these experiments demonstrate that in principle, individual synaptic terminals have the capacity to endocytose a large fraction of the recycling vesicle pool with a single time constant. In these experiments, the maximal $C_{\text {endo }}$ achieved is $\sim 60 \%$ of the recycling pool.

\section{Increasing the temperature to $36^{\circ} \mathrm{C}$ changes endocytic capacity and fundamental time constant}

We repeated the measurements of $\tau$ versus surface accumulation at $36^{\circ} \mathrm{C}$ to determine the behavior of synapses with respect to these basic endocytic parameters under more physiological conditions. Direct comparison of endocytosis at the same synapses after $150 \mathrm{AP}$ at $30 \mathrm{~Hz}$ showed that the time constant decreases from $\sim 14 \mathrm{~s}$ at room temperature to $\sim 6 \mathrm{~s}$ at $36^{\circ} \mathrm{C}$ (Fig. $7 A)$. Similar results were obtained for 15 AP stimulation at $30 \mathrm{~Hz}$, suggesting that the fundamental time constant is $\sim 6 \mathrm{~s}$. In addition, we determined the time constant 
for vesicle reacidification using the approach described previously (Atluri and Ryan) and determined that at $36^{\circ} \mathrm{C}$, vesicle reacidification occurs with a time constant of $\sim 2.3 \mathrm{~s}$ (data not shown). In agreement with previous measurements (Pyott and Rosenmund, 2002; Fernandez-Alfonso and Ryan, 2004; Klyachko and Stevens, 2006), the rate of action potential-driven exocytosis for repeated stimulation decreases as the temperature increases (Fig. $7 A$ ). This is thought to result in part from the fact that with each stimulus, fewer calcium ions enter into the nerve terminal, because of the narrowing of the action potential, as well as from the increase in the kinetics of calcium clearance. The significantly faster value of $\tau_{0}$ combined with the decrease in exocytosis rates both result in lower total accumulation of synaptic com-

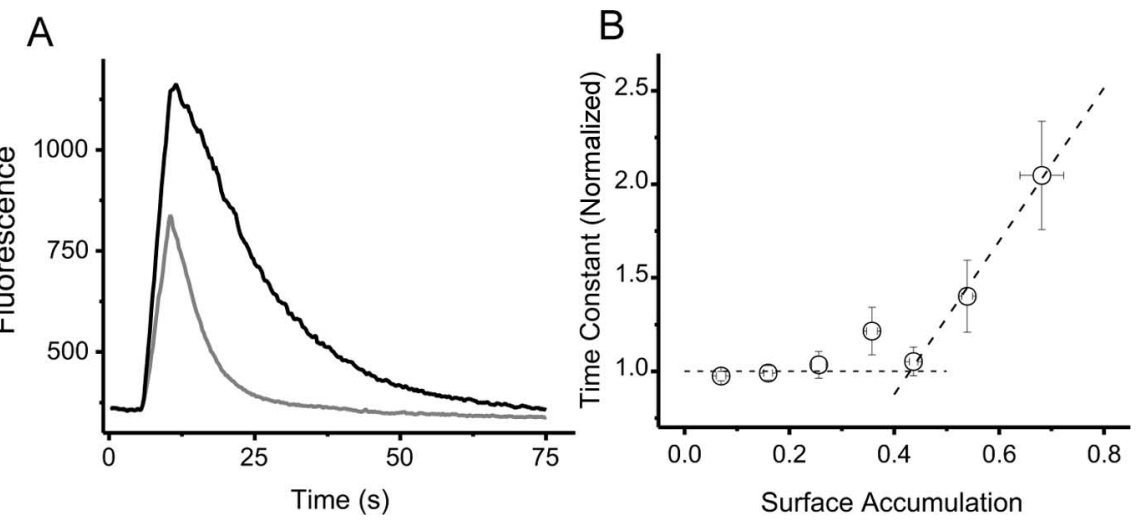

Figure 7. Fundamental time constant is faster at $36^{\circ} \mathrm{C}$. $\boldsymbol{A}$, Comparison of the fluorescence signal after a $150 \mathrm{AP}$ stimulus for the same set of 49 boutons at $28^{\circ} \mathrm{C}$ (black), which yields $\tau=14.96 \pm 0.08$, and at $36^{\circ} \mathrm{C}$ (gray), yielding $\tau=5.79 \pm 0.04 . \boldsymbol{B}$, Saturation of the endocytic capacity is apparent at $36^{\circ} \mathrm{C}$ during $30 \mathrm{~Hz}$ stimulation. Synapses were stimulated with varying numbers of $\mathrm{AP}(15-450)$ at $30 \mathrm{~Hz}$, and the time constants for $\mathrm{vGpH}$ decays were obtained for each accumulation level. These data were obtained from 10 cells (31-93 boutons each), and the time constants for a given cell were normalized to the smallest value in the nonsaturating region of each cell. The average time constant in the nonsaturating region is $6 \pm 1.3 \mathrm{~s}$. ponents on the cell surface during stimulus

trains, in agreement with our previous measurements (Fernandez-Alfonso and Ryan, 2004). To examine the potential impact of endocytic capacity on endocytosis, we therefore used higher-frequency stimulation where more significant accumulation occurs. Repeated trials for $30 \mathrm{~Hz}$ stimulation with varied number of APs showed very similar behavior to that seen at room temperature. Below a critical level of accumulation, the time constant for endocytosis remained invariant, and above this value it increased approximately linearly. The value of $C_{\text {endo }}$ at $36^{\circ} \mathrm{C}$, however, is somewhat lower at the more elevated temperature, occurring at $\sim 45 \%$ of the vesicle pool compared with $\sim 60 \%$ for these higher frequency stimuli at room temperature. The slight shift to lower values is consistent with the fact the accumulation of intracellular calcium is significantly decreased at this temperature (Atluri and Regehr, 1996).

\section{Discussion}

The ability to recycle synaptic vesicles is a critical property of nerve terminals, whose molecular basis has been under intense investigation for the last decade. During repetitive stimulation, synaptic vesicle components accumulate on the synaptic surface, as has been shown by both electrical capacitance measurements (von Gersdorff and Matthews, 1994a; Neves et al., 2001; Wu et al., 2005; Yamashita et al., 2005) and electron microscopy in giant synapses (Shupliakov et al., 1997), as well as with pHluorintagged vesicle components in small CNS nerve terminals (Sankaranarayanan and Ryan, 2000; Li and Murthy, 2001) and at neuromuscular junctions (Tabares et al., 2007). We operationally defined the endocytic capacity of the synapse as the maximal number of vesicles that can be endocytosed with an invariant endocytic characteristic time, and have taken advantage of improvements in the sensitivity of pHluorin-based assays to examine how the endocytosis time constant depends on the total accumulation of synaptic vesicle components over a wide range of stimulation level. Our data reveal that the characteristic time for endocytic recovery is invariant for stimuli between 1 and 100 AP at $10 \mathrm{~Hz}$ or above for $\mathrm{spH}$ as well as $\mathrm{vGpH}$, and is identical to that for retrieving single vesicles (Balaji and Ryan, 2007). We thus refer to the time constant in this regime as the fundamental time constant, $\tau_{0}$, because it corresponds to the retrieval time for individual vesicles. Our experiments revealed that the time constant for recovery was not sensitive to the conditions of stimulation, provided the total accumulation of vesicle components remained below a critical value. This was true even during trains of repetitive stimulation, where, to determine the average time constant for endocytosis, we developed a novel approach that examines rates of exocytosis and net accumulation simultaneously for stimulus frequencies between 0.05 and $1 \mathrm{~Hz}$. Under these conditions, the average time constant was statistically indistinguishable from the value of $\tau_{0}$ obtained for recovery from brief stimuli. Conditions that led to greater intracellular calcium accumulation, either by increasing the stimulus frequency or by stimulating in the presence of elevated external calcium ion concentration, expanded the range over which the time constant remained invariant, thus revealing an adaptive response that increases the value of $C_{\text {endo. }}$. This increase in capacity results in an overall increase in the rate of endocytosis, in agreement with previous studies both in hippocampal synapses and at the calyx of Held.

The role of intracellular calcium in endocytosis at nerve terminals has been investigated for a number of synapses (von Gersdorff and Matthews, 1994b; Neves et al., 2001; Sankaranarayanan and Ryan, 2001; Wu et al., 2005). Early studies at the frog neuromuscular junction showed that prolonged stimulation with black widow venom in the absence of calcium led to a profound depletion of synaptic vesicles at the nerve terminal (Ceccarelli and Hurlbut, 1980). This result led to the hypothesis that elevation in intracellular calcium played an essential role in synaptic vesicle endocytosis. However, more recent studies have examined endocytosis in response to acute manipulations of intracellular calcium during exocytosis. In general, elevation in intracellular calcium leads to acceleration in endocytic rates. In bipolar cells, endocytosis appears to occur via two pathways that differ in timescale by $\sim 1$ order of magnitude (Neves et al., 2001). In those synapses, elevation in intracellular calcium appears to preferentially shift endocytic recovery to the faster of the two pathways [although see von Gersdorff and Matthews (1994b), which showed that complete inhibition of endocytosis can also be achieved with sufficient calcium elevation]. At the calyx of Held, the acceleration has been attributed to the appearance of a faster pathway driven by calcium, which leads to an increased overall rate (Wu et al., 2005). At the small synapses typical of the CNS that were studied here, our data are consistent with a model whereby modulation by calcium is achieved at least in part by simply expanding the capacity of a process that has previously 
been shown to be clathrin dependent (Granseth et al., 2006). By inference, this implies that calcium is impacting the number of functional sites for endocytosis. These results suggest that rather than playing an essential role, elevation of calcium plays a modulatory role. If sufficient exocytosis can be achieved in the absence of calcium elevation, the resulting endocytosis could appear to be impaired, as in the case of the latrotoxin stimulation at the neuromuscular junction; however, this result may simply reflect a profound imbalance in the rates of exocytosis versus endocytosis, which would occur if the rate of assembly of endocytic sites was kept very low. This proposed role of calcium in endocytosis is also in agreement with results obtained in the giant reticulospinal synapse in the lamprey (Gad et al., 1998), where it was possible to temporally dissociate the calcium influx that occurs during stimulation from endocytic recovery.

At the molecular level, a number of endocytic proteins, referred to as dephosphins, have been shown to be substrates for the calciumdependent phosphatase calcineurin (Cousin and Robinson, 2001). Included in this family are dynamins, amphiphysin, and synaptojanin, all of which become dephosphorylated at nerve terminals during activity. The precise role of these dephosphorylation events has not been elucidated in most cases, although expression of phosphomimetic mutants of dynamin 1 show strong dominant-negative effects on vesicle recycling (Anggono et al., 2006), and these studies show that dephosphorylation of dynamin 1 allows it to bind another endocytic protein, syndapin. Experiments using pharmacological or molecular intervention of the phosphatase and kinase thought to control the phosphorylation state of dephosphins have indicated that endocytosis can be slowed via these interventions (Evans and Cousin, 2007). Given the strong correlation we find between intracellular calcium levels and the endocytic capacity at individual synapses, we speculate that calcium, through the action of calcineurin and dephosphorylation of critical endocytic proteins, promotes more rapid assembly of the endocytic machinery, allowing a greater number of endocytic sites to become functional during repetitive stimulation. Our studies show that elevation of intracellular calcium, which occurs during repetitive stimulation, does not alter the fundamental time constant for an individual vesicle's endocytosis. This was true both for modest stimulation frequency, where it was possible to derive the mean rate constant during steady-state firing, and after high-frequency bursts.

In contrast, elevation of the temperature from room temperature to $36^{\circ} \mathrm{C}$ resulted in a decrease in $\tau_{0}$ by more than twofold. This higher rate constant for endocytosis in turn means that during repetitive stimulation, fewer vesicles accumulate on the synaptic surface, and the endocytic capacity is only exceeded during prolonged high-frequency bursts. At physiological temperature, the total endocytic capacity for stimulation at $30 \mathrm{~Hz}$ is $\sim 45 \%$ of the recycling pool.

Direct optical quantal analysis in these synapses indicated that the total recycling pool comprises $\sim 64$ vesicles for these synapses (Balaji and Ryan, 2007). This calibration allows us to determine the value of $C_{\text {endo }}$ in terms of the number of vesicles being endocytosed in parallel at each synapse. At $36^{\circ} \mathrm{C}$, this corresponds to $\sim 28$ vesicles; however, the system seems capable of even greater capacity ( $\sim 38$ vesicles) when sufficient calcium accumulation occurs (at high frequency at room temperature).

\section{A model incorporating a single mode of endocytosis with variable and calcium-controlled capacity}

The primary observation in our dataset is that the time course of endocytosis is well described by a single exponential process whose time constant is invariant for a wide range of surface accumulation of synaptic vesicles. This basic phenomenology implies but does not prove that a single molecular process operates over this entire regime of constant retrieval time scale, and for simplicity, we have defined the maximal accumulation for which the time constant for retrieval does not change as the endocytic capacity of the synapse. Our observations that manipulations that lead to variations in the steady-state calcium during stimulation alter the endocytic capacity lead us to propose that this capacity is defined by the rate at which endocytic machinery can be assembled, and that this rate is in turn controlled by calcium. After stimulation periods, calcium typically decays within $\sim 5 \mathrm{~s}$ (Fig. $6 A$ ), and thus we would expect that the rate of assembly would significantly decrease during this period. If the total amount of exocytosis exceeds the number of sites that could be assembled, then retrieval time slows. A detailed model explaining exactly how much slower it should become is beyond the reach of the current study, because it would require a more detailed knowledge of how quickly sites can be rebuilt after calcium has decayed. Evans and Cousin (2007) have proposed an alternate view of vesicle recycling whereby only the poststimulus retrieval would operate via machinery that uses dephosphins. In this view, the increase in $C_{\text {endo }}$ we see could be explained by an increase in the efficiency of endocytosis in the poststimulus period. However, given that we found that endocytosis during stimulation strictly requires dynamin (Ferguson et al., 2007), we favor the view that dephosphins are operational over the entire range of stimuli.

In conclusion, our data reveal that modulation of endocytic machinery provides an adaptive mechanism for the synapse to handle the greater endocytic loads that result during high-frequency firing and has identified two potential points of modulation. One is the endocytic capacity, the other the fundamental time constant that corresponds effectively to the time required to endocytose individual vesicles. These experiments have provided a first quantitative estimate of the number of endocytic events that can occur in parallel at individual synapses, and indicate that the intrinsic endocytic capacity of these synapses is very high. The ability to tap into this intrinsic ability is mediated by elevation in intracellular calcium, and thus the endocytic capacity is a dynamic property of the synapse. Our studies reveal that at physiological temperature in the CNS synapses studied here, accumulation of vesicle components exceeds the capacity only during prolonged stimulation at high frequency, and therefore in most cases the total retrieval time after stimulation is on the order of $\sim 6 \mathrm{~s}$.

\section{Appendix}

Kinetics of endocytosis and reacidification

$$
R(t) \stackrel{k_{x}}{\rightarrow} S(t) \stackrel{k_{n}}{\rightarrow} A(t) \stackrel{k_{r}}{\rightarrow} D(t)
$$

To estimate the effects of reacidification on our fluorescence measurement of endocytosis, we developed a sequential firstorder reaction model, as illustrated above. $R(t), S(t)$, and $A(t)$ are continuous approximations of vesicle counts of releasable, surface alkaline, and internal alkaline pool as a function of time. Assuming a first-order reaction with rate constants $k_{x}, k_{n}$, and $k_{r}$ for exocytosis, endocytosis, and reacidification, the rate law for the above reaction is as follows:

$$
\begin{aligned}
& R^{\prime}=-k_{x} R \\
& S^{\prime}=k_{x} R-k_{n} S \\
& A^{\prime}=k_{n} S-k_{r} A, \text { where } f^{\prime} \text { is } \frac{d f}{d t}
\end{aligned}
$$


Fluorescence $F(t) \propto S(t)+A(t)$.

Solving for $F(t)$, we get

$$
\begin{aligned}
& F(t)=\alpha \Psi_{0} \phi e^{-k_{n} t}+\frac{\phi \Psi_{0} k_{x} e^{-k_{x} t}}{\left(k_{x}-k_{n}\right)}\left(e^{-\left(k_{n}-k_{x}\right) t}-1\right) \\
& +\frac{\phi \Psi_{o} k_{n} k_{x} e^{-k_{r} t}}{\left(k_{x}-k_{n}\right)} \cdot\left(\frac{\left(e^{-\left(k_{n}-k_{r}\right) t}-1\right)}{k_{r}-k_{n}}-\frac{\left(e^{-\left(k_{x}-k_{r}\right) t}-1\right)}{k_{r}-k_{x}}\right),
\end{aligned}
$$

where $\alpha$ is the surface fraction, $\Psi_{0}$ is pool size, and $\phi$ is the number of fluorescence counts per molecule of GFP. Equation 2 describes the time evolution of the fluorescence for small stimuli that avoid depleting the pool: $t \ll 1 / k_{x}, 1 / k_{r}, 1 / k_{n}$. The region where the rate of exocytosis equals the rate of endocytosis is identified by when the fluorescence attains its maximal value $\left(F_{\max }\right)$, $d F(t) / d t \approx 0$. This region is seen in Figure $3 B$ when the endocytosis and exocytosis curves are parallel.

The curve

$$
\begin{aligned}
E^{T}(t) & =\Psi_{0}-F(t)-S(t) \\
& =\Psi_{0}-R(t)-F(t)+A(t)
\end{aligned}
$$

represents the endocytosis time trace.

$$
E^{T}=E^{A}+A(t)
$$

$E^{A}$ is constructed by subtracting the fluorescence curve from the exocytosis curve using Equation $3 b$. The exocytosis curve is constructed from the fluorescence traces of Figure $3 A$ by plotting only the exocytic transitions. Our measurable quantities are the endocytosis curve, $E^{A}$, the exocytosis curve, Ex, and the fluorescence, $F(t)$.

At $F_{\text {max }}$, we have

$$
\frac{d F}{d t}=0 \Rightarrow \frac{d E^{T}}{d t}=-\frac{d R}{d t} \equiv \frac{d E x}{d t} .
$$

Using Equations 5, 4, 3, and 1,

$$
\begin{aligned}
\tau_{n}=\frac{1}{k_{n}} & =\frac{S(t)}{d E^{T} / d t} \mid \frac{d F}{d t}=0 \\
& =\frac{f(t)}{d E^{A} / d t} \mid \frac{d F}{d t}=0-\frac{1}{k_{r}} .
\end{aligned}
$$

To measure the endocytosis time constant, we construct the apparent endocytosis curve according to Equation 4. We measure the endocytosis time constant by measuring the slope of the apparent endocytosis curve and using it in Equation 6 along with steady-state fluorescence and reacidification time constant.

\section{The poststimulus decay}

From equations 2 and 1 above, it can be shown that for the poststimulus region setting $\left[k_{x}=0\right.$ and solving for $S(t)$ and $\left.A(t)\right]$, we get

$$
F(t)=\frac{\Psi_{0} \phi \tau_{n} e^{-\frac{t}{\tau_{n}}}}{\left(\tau_{n}-\tau_{r}\right)}-\frac{\Psi_{0} \phi \tau_{r} e^{-\frac{t}{\tau_{r}}}}{\left(\tau_{n}-\tau_{r}\right)},
$$

where $\tau_{x}, \tau_{n}$, and $\tau_{r}$ are time constants for exocytosis, endocytosis, and reacidification. For times $t>\tau_{r}$, the expression in Equation 7 reduces to a single exponential.

\section{References}

Anggono V, Smillie KJ, Graham ME, Valova VA, Cousin MA, Robinson PJ (2006) Syndapin I is the phosphorylation-regulated dynamin I partner in synaptic vesicle endocytosis. Nat Neurosci 9:752-760.
Atluri PP, Regehr WG (1996) Determinants of the time course of facilitation at the granule cell to Purkinje cell synapse. J Neurosci 16:5661-5671.

Atluri PP, Ryan TA (2006) The kinetics of synaptic vesicle reacidification at hippocampal nerve terminals. J Neurosci 26:2313-2320.

Balaji J, Ryan TA (2007) Single-vesicle imaging reveals that synaptic vesicle exocytosis and endocytosis are coupled by a single stochastic mode. Proc Natl Acad Sci USA 104:20576-20581.

Ceccarelli B, Hurlbut WP (1980) Ca2+-dependent recycling of synaptic vesicles at the frog neuromuscular junction. J Cell Biol 87:297-303.

Cousin MA, Robinson PJ (2001) The dephosphins: dephosphorylation by calcineurin triggers synaptic vesicle endocytosis. Trends Neurosci 24:659-665.

Di Paolo G, Moskowitz HS, Gipson K, Wenk MR, Voronov S, Obayashi M, Flavell R, Fitzsimonds RM, Ryan TA, De Camilli P (2004) Impaired PtdIns(4,5)P2 synthesis in nerve terminals produces defects in synaptic vesicle trafficking. Nature 431:415-422.

Evans GJ, Cousin MA (2007) Activity-dependent control of slow synaptic vesicle endocytosis by cyclin-dependent kinase 5. J Neurosci 27:401-411.

Ferguson SM, Brasnjo G, Hayashi M, Wolfel M, Collesi C, Giovedi S, Raimondi A, Gong LW, Ariel P, Paradise S, O’Toole E, Flavell R, Cremona O, Miesenbock G, Ryan TA, De Camilli P (2007) A selective activity-dependent requirement for dynamin 1 in synaptic vesicle endocytosis. Science 316:570-574.

Fernandez-Alfonso T, Ryan TA (2004) The kinetics of synaptic vesicle pool depletion at CNS synaptic terminals. Neuron 41:943-953.

Gad H, Low P, Zotova E, Brodin L, Shupliakov O (1998) Dissociation between Ca2+-triggered synaptic vesicle exocytosis and clathrin-mediated endocytosis at a central synapse. Neuron 21:607-616.

Granseth B, Odermatt B, Royle SJ, Lagnado L (2006) Clathrin-mediated endocytosis is the dominant mechanism of vesicle retrieval at hippocampal synapses. Neuron 51:773-786.

Klyachko VA, Stevens CF (2006) Excitatory and feed-forward inhibitory hippocampal synapses work synergistically as an adaptive filter of natural spike trains. PLoS Biol 4:e207.

Li Z, Murthy VN (2001) Visualizing postendocytic traffic of synaptic vesicles at hippocampal synapses. Neuron 31:593-605.

Mani M, Lee SY, Lucast L, Cremona O, Di Paolo G, De Camilli P, Ryan TA (2007) The dual phosphatase activity of synaptojanin 1 is required for both efficient synaptic vesicle endocytosis and reavailability at nerve terminals. Neuron 56:1004-1018.

Neves G, Gomis A, Lagnado L (2001) Calcium influx selects the fast mode of endocytosis in the synaptic terminal of retinal bipolar cells. Proc Natl Acad Sci USA 98:15282-15287.

Pyott SJ, Rosenmund C (2002) The effects of temperature on vesicular supply and release in autaptic cultures of rat and mouse hippocampal neurons. J Physiol 539:523-535.

Ryan TA, Smith SJ (1995) Vesicle pool mobilization during action potential firing at hippocampal synapses. Neuron 14:983-989.

Sankaranarayanan S, Ryan TA (2000) Real-time measurements of vesicleSNARE recycling in synapses of the central nervous system. Nat Cell Biol 2:197-204.

Sankaranarayanan S, Ryan TA (2001) Calcium accelerates endocytosis of vSNAREs at hippocampal synapses. Nat Neurosci 4:129-136.

Sankaranarayanan S, De Angelis D, Rothman JE, Ryan TA (2000) The use of pHluorins for optical measurements of presynaptic activity. Biophys J 79:2199-2208.

Schweizer FE, Ryan TA (2006) The synaptic vesicle: cycle of exocytosis and endocytosis. Curr Opin Neurobiol 16:298-304.

Shupliakov O, Low P, Grabs D, Gad H, Chen H, David C, Takei K, De Camilli P, Brodin L (1997) Synaptic vesicle endocytosis impaired by disruption of dynamin-SH3 domain interactions. Science 276:259-263.

Tabares L, Ruiz R, Linares-Clemente P, Gaffield MA, Alvarez de Toledo G, Fernandez-Chacon R, Betz WJ (2007) Monitoring synaptic function at the neuromuscular junction of a mouse expressing synaptopHluorin. J Neurosci 27:5422-5430.

von Gersdorff H, Matthews G (1994a) Dynamics of synaptic vesicle fusion and membrane retrieval in synaptic terminals. Nature 367:735-739.

von Gersdorff H, Matthews G (1994b) Inhibition of endocytosis by elevated internal calcium in a synaptic terminal. Nature 370:652-655.

Wu W, Xu J, Wu XS, Wu LG (2005) Activity-dependent acceleration of endocytosis at a central synapse. J Neurosci 25:11676-11683.

Yamashita T, Hige T, Takahashi T (2005) Vesicle endocytosis requires dynamin-dependent GTP hydrolysis at a fast CNS synapse. Science 307: $124-127$. 